\title{
Correspondence analysis method of anti-slide pile information monitoring and slice model
}

\author{
Wanqi Wang* \\ China Academy of Railway Sciences Corporation Limited Institute of Computing Technology, Beijing, 100081 China
}

\begin{abstract}
In order to effectively grasp the real-time working status of anti-slip piles and the reinforcement effect of slopes, timely information monitoring of anti-slip piles is carried out, and the reinforcement range is modeled, and the analysis and evaluation method of slicing model numerical calculation and information monitoring data is proposed, and the information monitoring data is compared with numerical simulation to achieve effective grasp of By comparing the information monitoring data with the numerical simulation, we can effectively grasp the timely working condition of the anti-slip pile and the reinforcement effect of the soil around the pile. This is a very important engineering practice for the evaluation of anti-slip pile reinforced slope areas.
\end{abstract}

Keywords: anti-slip piles; information monitoring; numerical model; evaluation

\section{Introduction}

Lei Wenjie et al [1] conducted numerical simulation of slope reinforced by anti-slip piles based on the finite element strength reduction method, and explored the influence of pile position on the stability safety coefficient, location and shape of the most dangerous sliding surface of the slope The relationship between the pile spacing and the friction angle within the soil as well as the pile spacing and the slope inclination was analyzed by Zhao Minghua et al [2] based on the sloping arch effect of the soil between piles; Nian Yankai et al $[3,4]$ used the upper and lower limit theorems of plastic limit analysis to analyze the anchorage depth and the ultimate earth pressure on the pile side of the anti-slip pile considering the influence of various parameters in the slope; Ausilio et al [5] obtained the relationship between the optimal position of anti-slip piles and the slope safety factor by analyzing the stability of slopes with and without piles, and determined the critical sliding surface and safety factor (Eq.) for slopes without piles; Hassiotis et al [6] performed stability analysis of slopes based on the friction circle method, and introduced the stability number considering anti-slip piles to determine the new most dangerous sliding surface and safety factor; Won [7] used a shell unit simulated the interaction relationship between the anti-slip pile and the soil body, obtained the safety coefficient of the slope under different pile stiffness and pile head restraint conditions, and analyzed the shear force on the contact surface, and proposed the location of the point corresponding to the slip crack surface at the pile setting at the maximum shear force of the pile body; Dai Zihang [8] studied and deduced the different distribution of the landslide thrust and soil and rock resistance based on the model test of anti-slip pile and field test results in China functions; Song Yingjie et al [9] reviewed the theories of domestic and foreign methods on stability analysis of antislip column reinforced slopes and compared and analyzed their advantages and disadvantages; Liu Bo et al [10] used FLAC $\sim(3 \mathrm{D})$ for numerical simulation to study the mechanism of anti-slip pile overtopping failure of a high slope under rainfall conditions and made trial calculations on the reasonable location of anti-slip piles; Liu Tao et al [11] constructed a three-dimensional multilayered landslide body model, analyzed the formation process of the soil arch behind the pile and the four stages of soil arch evolution, and proposed a strip division method applicable to multilayered landslides.Zhang Youjun et al.[12] analyzed the various characteristics of landslides during the sliding, sliding acceleration and sliding mitigation stages through the observation data of landslide surface and support structure (anti-sliding pile) displacement, anti-sliding pile stress monitoring, landslide body deep displacement monitoring, groundwater level monitoring and fracture inspection. The monitoring methods complement each other and provide a comprehensive and accurate understanding of the state of the landslide body, which provides a reliable basis for the information design and safe construction of landslide management. By monitoring the underground displacement during the construction of the landslide, Long Wanxue et al.[13] used hyperbolic exponential smoothing, BP neural network and polynomial regression for tracking prediction, and Verhulst inverse function model for time prediction, analyzed the sliding speed and deformation stage of the landslide, combined with rainfall

\footnotetext{
* Corresponding author: wangwanqi@rails.cn
} 
and construction for comprehensive analysis, dynamically adjusted the construction organization plan, and took effective measures in time, The construction organization plan was dynamically adjusted and effective measures were taken in time to ensure the safety of the project.

The above study discussed the influence of anti-slip piles on slope stability and the influence of anti-slip pile parameter settings on slope stability, but there is no analysis method for the integration of numerical simulation technology and information monitoring technology items.

\section{Structural slice model}

\subsection{Slope slicing model}

The slope model can be simulated based on the topographic map of the slope at the site, import the digital map, and then slice the location of the less stable areas for slope stability analysis, so as to determine the location information where anti-slip piles or other measures for reinforcement are needed.

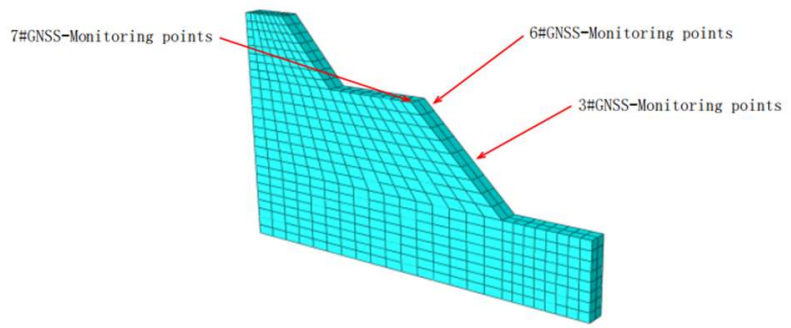

Figure 1 Slope finite element model and monitoring point arrangement

\subsection{Slice model under anti-slip pile reinforcement}

According to the previously selected reinforcement location, numerical modeling with different reinforcement depth, reinforcement spacing and other parameter variations is carried out, and the specific reinforcement location is determined after considering the economic analysis. After that, slice analysis is performed according to the reinforcement location of the pile to effectively grasp the timely working condition of the antislip pile and the reinforcement effect of the soil around the pile.

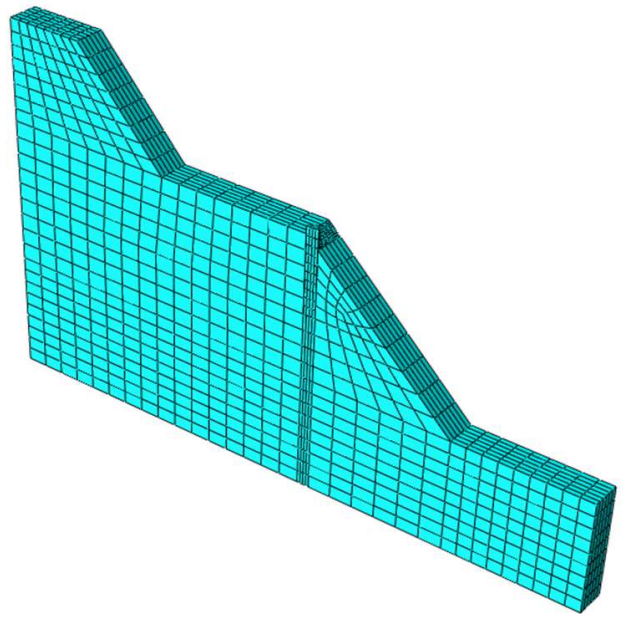

(a) Upper

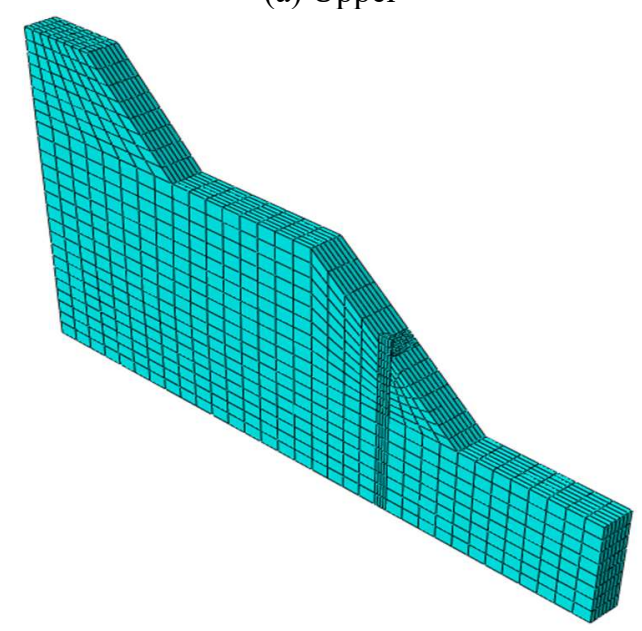

(b) Middle

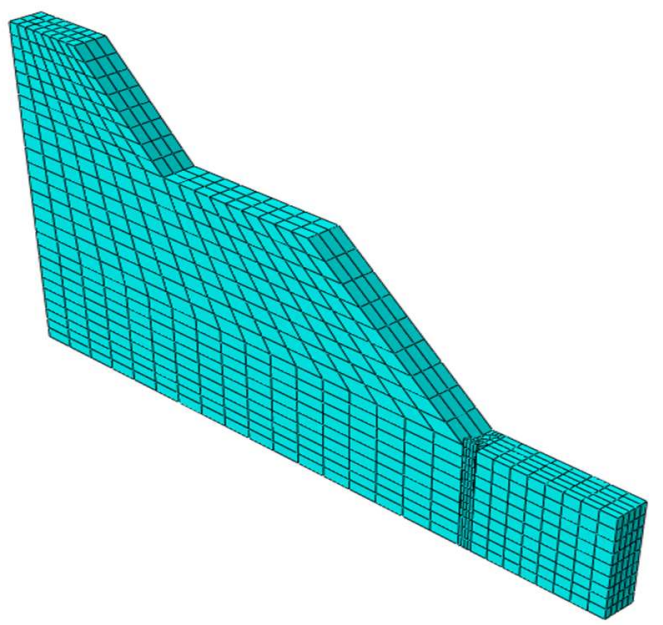

(c) Bottom 


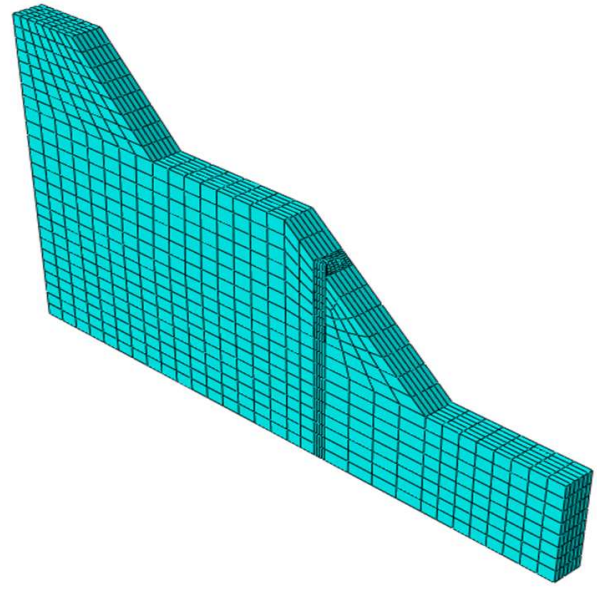

(d) Upper middle

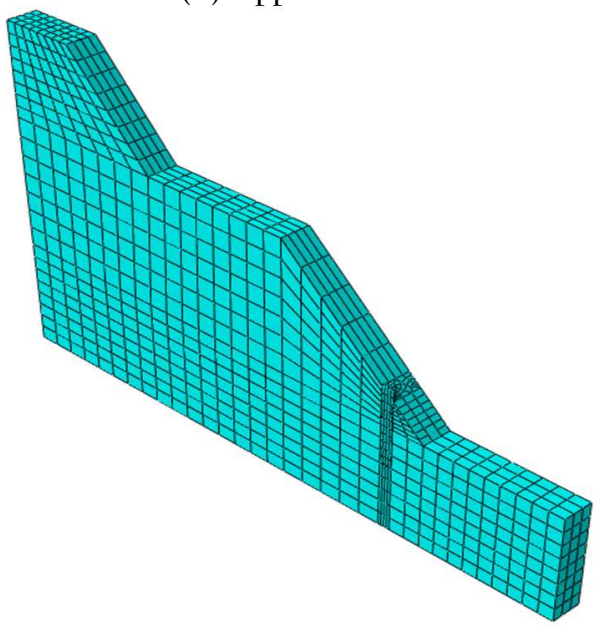

(e) Lower middle

Figure 2 Schematic diagram of anti-slip pile laying

\subsection{Numerical simulation results}

The displacement clouds of the slope and anti-slip piles when the anti-slip piles are set at the same location as the actual setting will be shown here as a basis for corresponding to the actual monitoring data in the following.
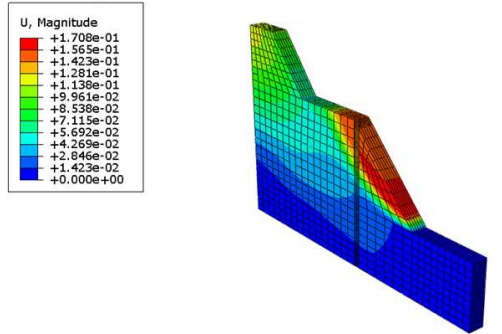

Figure 3 Numerical simulation results (Displacement cloud map)

\section{Informative Monitoring}

\subsection{Information monitoring of the working condition of anti-slip piles}

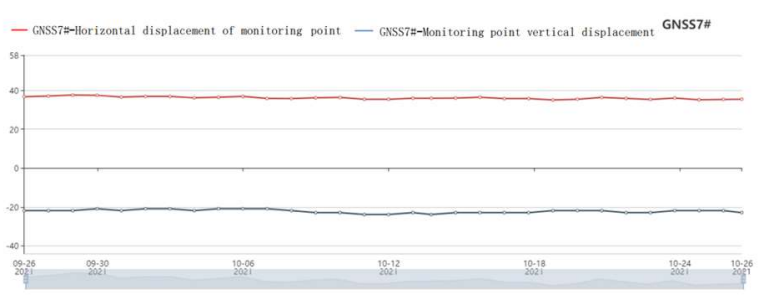

Figure 4 Monitoring point displacement data(GNSS7\#)

Point 7 is located on the outside of the anti-slip pile and the upper part of the landslide body, which is used to monitor the displacement of the lower part of the landslide body and the outside of the anti-slip pile.

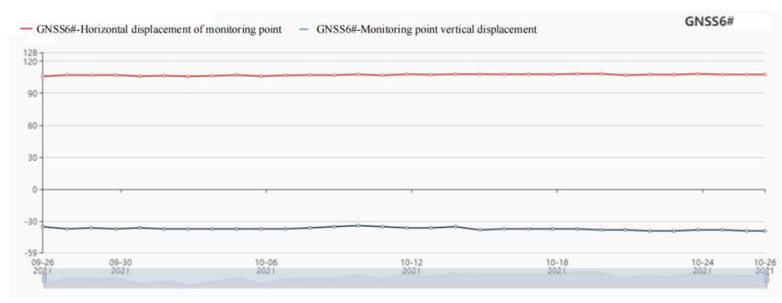

Figure 5 Monitoring point displacement data(GNSS6\#)

Point 6 is placed inside the anti-slip pile and the upper part of the landslide body, which is used to monitor the displacement of the upper part of the landslide body and the inner part of the anti-slip pile.

From the monitoring data of GNSS7\# and GNSS6\#, we can see that the horizontal displacement of $7 \#$ is around 37 40 and the vertical displacement is around -18 -23; the horizontal displacement of $6 \#$ is around 100 110 and the vertical displacement is around $-31 \sim-40$. Both monitoring points are in stable condition, and the monitoring data can correspond to the numerical simulation when the anti-slip pile is set in the upper part, as shown in Figure 3.

\subsection{Information monitoring of key slope locations}

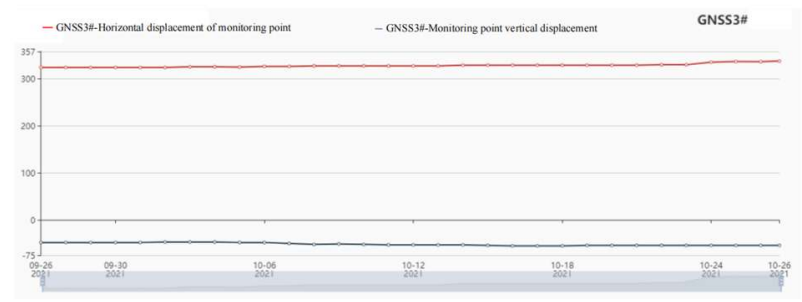

Figure 6 Monitoring point displacement data(GNSS3\#)

Point 3 GNSS is placed at the foot of the landslide body to monitor the lower displacement of the landslide body. 


\section{Conclusions}

The piles are monitored by information technology in time, and the reinforcement range is modeled, and an analysis and evaluation method is proposed for the numerical calculation of the slice model and the information technology monitoring data.

\section{References}

1. Lei Wenjie, Zheng Yinren, Feng Xiating. Analysis of anti-slip piles piling in landslide management[J]. Geotechnics,2006,27(6):950-954.

2. Zhao Minghua., Liao Binbin., Liu Sisi. Pile spacing calculation of anti-slip piles on slopes based on arch effect[J]. Geotechnics,2010,31(4):1211-1216.

3. Nian Tingkai, Luan Maotian, Zheng Defeng, et al. Limit analysis of the anchorage depth of antislip piles[J]. Journal of Water Resources,2007(6):743748.

4. Nian Tingkai, Luan Maotian, Zheng Defeng, et al. A simplified analysis method of anti-slip pile considering the effect of pore water pressure in slope[J]. Geotechnics,2008(4):1067-1071.

5. E. Ausilio,E. Conte,G. Dente. Stability analysis of slopes reinforced with piles[J]. Computers and Geotechnics,2001,28(8).

6. HASSIOTIS S,CHAMEAU J L, GUNARATNE M. Design methodfor stabilization of slopes piles[J].Journal of Geotechnical \&Geoenvironmental Engineering, 1997,125(10):314-323.

7. Jinoh Won,Kwangho You,Sangseom Jeong,Sooil Kim. Coupled effects in stability analysis of pileslope systems[J]. Computers and Geotechnics,2005,32(4).

8. Dai Zihang. Study on the distribution law of landslide thrust and pile anterior slide resistance of anti-slip piles[J]. Journal of Rock Mechanics and Engineering, 2002, 21(4):517-521.

9. Song Yinjie, Chen Wenqiang, Li Changdong. Progress of slope stability evaluation and pile optimization after anti-slip pile reinforcement[J]. Safety and Environmental Engineering, 2016,23(5):43-49.

10. Liu Bo, Su Peidong, Qiu Peng,et al. Numerical simulation of high-slope anti-slip pile overtopping problem[C].Proceedings of the 2016 National Academic Conference on Engineering Geology.2016.

11. Liu Tao, Zhang Haikuan, Zhang You, et al. Study on the minimum pile spacing of anti-slip piles in threedimensional composite multilayered landslides[J]. Journal of Rock Mechanics and Engineering, 2018, 37(2):473-484.

12. Zhang Youjun, Wan Champion, Li Yi. Research on the application of multiple monitoring tools in landslide monitoring $[\mathrm{J}]$.
13. Long Wanxue. Application of monitoring and forecasting technology in landslide information construction [A]. Guizhou Rock Mechanics and Engineering Society, Guiyang Survey and Design Institute of China Hydropower Consultants Group. Proceedings of the 2008 Annual Academic Conference of Guizhou Rock Mechanics and Engineering Society[C]. Guizhou Rock Mechanics and Engineering Society, Guiyang Survey and Design Institute of China Hydropower Consultants Group:Guizhou Science and Technology Association, 2008:4 